\title{
INFLUENCE OF LOCAL ZONES OF INTENSIVE HEAT TRANSFER ON THERMAL REGIME OF HEAT SUPPLY OBJECTS
}

\author{
Vyacheslav I. Maksimov, Tatiana A. Nagornova, OlgaV. Stepanova \\ Institute of Power Engineering, National research Tomsk Polytechnic University, Tomsk 634050, \\ Russia
}

\begin{abstract}
The results of mathematical simulation of conjugate heat transfer for heat supply object are represented. The turbulent regime of air motion in a closed cavity with the enclosing walls from reinforced concrete and glass is examined. On the outer boundary, which includes window aperture, the conditions of convective-radiation heat exchange with environment are realized. Is solved the system of the dimensionless equations within the framework of thermal conductivity model for the solid walls and Navier-Stokes for the gas. The influence of the local zones of intensive heat transfer on the thermal regime of the heat supply objects is established. Are determined the values of the dimensionless heat exchange coefficient on division border "air - wall". The analysis of the values of mean temperatures of the solution region is carried out.
\end{abstract}

\section{Introduction}

With the analysis of thermal conditions of the heating facilities and expenditures for heating in essence are used balance models [1], basis of which is assumption about the uniform temperature field of object. It is assumed with this approach that the gradients of temperatures both in air and in the enclosing constructions can be disregarded. It is at the same time known approach to the analysis of temperature pour on the objects of heat supply [2,3], whose basis - the solution of the problems of the combined heat transfer in the regime of air free convection and thermal conductivity in the enclosing constructions is similar [4,5].

The possibility much more detailed description of the temperature fields for various premises in models that take into account including the turbulent natural convection of air [2,3]. But results [2,3] are obtained for several idealized objects with the uniform conditions of heat sink on the boundaries of solution region. In the real practice such objects of heat supply are encountered more rarely than the having window apertures. The latter are characterized by properties substantially worse in comparison with the carrying monolithic constructions (brick, concrete or wooden) heatproof. Accordingly, the significant intensification of heat sink on the individual sections of the outer boundaries and reducting the temperature in all zones of heating facilities is possible. Up to now the influence of this factor specially was not investigated. 
Purpose of the work - mathematical simulation of the air free convection process in the typical object of heat supply taking into account heat sink on enclosing constructions and the heterogeneities of the thermal insulation properties under the conditions for the turbulent air motion.

\section{Physical and mathematical model}

Object of study is quite typical premise with the window aperture, equipped with one heating device (Fig. 1).

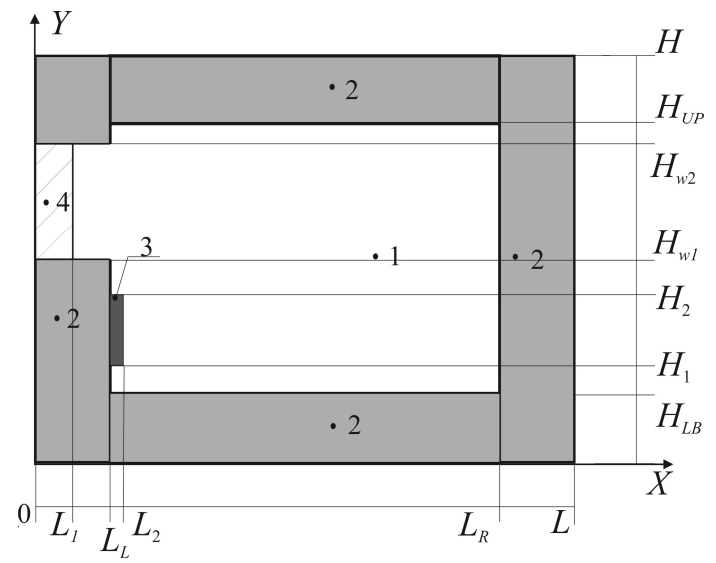

Figure 1. Area solution to the problem: 1 - air; 2 - enclosing structures; 3 - heat source; 4 - the window; $L$ - size of the area in the direction $X ; H$ - size of the solutions in the direction of $Y$; Indexes: $L, R, L B, U P$ - respectively left, right, upper and lower boundaries between media.caption.

In the longitudinal section the solution domain is a rectangle. Air quality is limited by the contourconducting walls of reinforced concrete and glass. On the left outside of the enclosing structure provided heat sink to the environment by convection and radiation. The heat sink is absent on the remaining three boundaries. On all conjugate boundaries "heat-conducting wall - air" accepted the conditions of the equality of heat fluxes and temperatures (boundary conditions of the fourth kind). On the surface heat source (heater) was set temperature (boundary conditions of the first kind). As the main mechanism of heat transfer in the system adopted for the thermal conductivity of walling and convection in the air region. The problem was solved in the conjugate formulation. Air flow described by differential Navier-Stokes equations in the Boussinesq approximation [4,5].

The system of equations describing the conjugate heat transfer in the system has the form:

$$
\begin{aligned}
& \frac{1}{\mathrm{Sh}} \frac{\partial \Omega}{\partial \tau}+U \frac{\partial \Omega}{\partial X}+V \frac{\partial \Omega}{\partial Y}=\frac{\partial^{2}}{\partial X^{2}}\left[\left(\frac{1}{\sqrt{\mathrm{Gr}}}\right) \Omega\right]+\frac{\partial^{2}}{\partial Y^{2}}\left[\left(\frac{1}{\sqrt{\mathrm{Gr}}}\right) \Omega\right]+\frac{1}{2} \frac{\partial \Theta}{\partial X}, \\
& \frac{\partial^{2} \Psi}{\partial X^{2}}+\frac{\partial^{2} \Psi}{\partial Y^{2}}=-2 \Omega, \\
& \frac{1}{\mathrm{Sh}} \frac{\partial \Theta}{\partial \tau}+U \frac{\partial \Theta}{\partial X}+V \frac{\partial \Theta}{\partial Y}=\frac{\partial}{\partial X}\left[\left(\frac{1}{\operatorname{Pr} \sqrt{\mathrm{Gr}}}\right) \Theta\right]+\frac{\partial}{\partial X}\left[\left(\frac{1}{\operatorname{Pr} \sqrt{\mathrm{Gr}}}\right) \Theta\right], \\
& \frac{1}{F_{0}} \frac{\partial \Theta}{\partial \tau}=\frac{\partial^{2} \Theta}{\partial X^{2}}+\frac{\partial^{2} \Theta}{\partial Y^{2}},
\end{aligned}
$$

Initial conditions for the system of equations (1-4):

$$
\Psi(X, Y, 0)=0, \quad \Omega(X, Y, 0)=0, \quad \Theta(X, Y, 0)=0 .
$$

Boundary conditions:

- at the three external borders of region solutions

$$
\frac{\partial \Theta(X, Y, \tau)}{\partial n}=0,
$$

- On the left outer boundary: 


$$
\frac{\partial \Theta(X, Y, \tau)}{\partial X}=\mathrm{Bi} \cdot \Theta(X, Y, \tau)+\mathrm{Bi} \cdot\left(\Theta_{e}\right)++N \cdot\left[(\Theta(X, Y, \tau))^{4}-\left(\Theta_{e}\right)^{4}\right] .
$$

- On interfaces walling and gas, the following conditions:

$$
\begin{gathered}
\frac{\partial \Psi(X, Y, \tau)}{\partial n}=0, \frac{\partial \Theta_{1}(X, Y, \tau)}{\partial n}=\lambda_{1,2} \frac{\partial \Theta_{2}(X, Y, \tau)}{\partial n}, \\
\Theta_{1}(X, Y, \tau)=\Theta_{2}(X, Y, \tau) .
\end{gathered}
$$

- On the surface of the heater adopted boundary conditions of the first kind

$$
\Theta(X, Y, \tau)=1 \text {. }
$$

где $X, Y$ - dimensionless Cartesian coordinates; $\Theta$ - dimensionless temperature; $\Omega$ - dimensionless analog vorticity; Fo $=a t_{0} / L^{2}$ - Fourier number; $\mathrm{Gr}=g \beta L^{3}\left(T_{i t}-T_{0}\right) / v_{t}{ }^{2}$ - Grashof number; $g$ acceleration created by the mass forces, $\mathrm{m} / \mathrm{s}^{2} ; a$ - thermal diffusivity, $\mathrm{m}^{2} / \mathrm{s} ; \beta$ - thermal coefficient of volume expansion, $\mathrm{K}^{-1} ; \mathrm{Bi}=\alpha L / \lambda-\mathrm{Biot}$ number; $\alpha$ - coefficient of heat exchange between the external environment and the area under consideration solutions; $\mathrm{N}=\varepsilon \sigma L\left(T-T_{e}\right)^{3} / \lambda$ - Starck number; $\varepsilon-$ a reduced degree of blackness; $\sigma-$ Stefan-Boltzmann constant; $\operatorname{Pr}=v_{t} / a-$ Prandtl number; $T-$ temperature, $\mathrm{K} ; T_{0}$ - temperature at the initial time, $\mathrm{K} ; T_{i t}$ - scale of temperature, $\mathrm{K} ; V_{0}$ - velocity scale (convection velocity), $\mathrm{m}^{2} / \mathrm{s} ; U, V$ - dimensionless velocity; $t$ - time, $\mathrm{s} ; t_{0}$ - time scale, $\mathrm{S} ; \lambda$ - thermal conductivity coefficient of solid wall, $\mathrm{W} /(\mathrm{m} \cdot \mathrm{K}) ; \lambda_{1,2}$ - relative thermal conductivity coefficient; $\mathrm{v}_{t}-$ coefficient of turbulent kinematic viscosity, $\mathrm{m}^{2} / \mathrm{s} ; \tau$ - dimensionless time; $\Psi$ - dimensionless analog stream function.

For solving the problem (1) - (9) algorithm was used [4, 5], developed for the numerical solution of natural convection in a closed rectangular regions with local sources of energy. Allowance for the influence of turbulence was conducted in the framework of algebraic Prandtl model [6]:

$$
v_{t}=l_{m}^{2}\left|\frac{\partial \bar{v}_{x}}{\partial y}\right|, l_{m}=k \cdot y,
$$

where $l_{m}$ - way of mixing, $v_{t}$ - dynamic coefficient of turbulent viscosity, $\mathrm{m}^{2} / \mathrm{s} ; k$ - universal constant of proportionality is independent of the Reynolds number.

\section{Analysis of numerical simulation results}

The following values of dimensionless temperatures were used for the realization of stated problem: heater $-\Theta_{i t}=1$, initial $-\Theta_{0}=0$, environment $-\Theta_{e}=-0.1$. The significant dimension of region $(6 \mathrm{~m})$ was selected on the basis of estimation of parameters of heat supply real objects.

Fig. 2 shows the results of numerical solution of the problem for a relatively small time interval (1 hour of physical time). Dynamics of change of temperature fields and contours stream function it makes it possible to isolate the convective air flows heated by source of heat emission. In the initial period of time (Fig. 2a-d) formed one large vortex, occupying all the space under consideration and insignificant turbulence near the source of the heat emissions, caused by the transiency of process and by intensive heat sink through the window aperture. With increasing time is separated into two distinct subdomains (Fig. 2e-h). It should be noted that window aperture initiates the formation of the vortex flows in immediate proximity from it.

The dynamics of a change in the average coefficient of dimensionless heat exchange ( $\mathrm{Nu}$ ) (especially from the surface of heater) characterizes nonmonotonic variation Nusselt number as a result of the transiency of process and corresponding transformation temperature fields (Fig. 3). Nevertheless after $\tau=70000$ it is noticeable (Fig. 3, 4) tendency toward the stabilization $\mathrm{Nu}$ (with exception of the dependence, which characterizes heat emission from the surface of heater). 
Based on the (Fig. 3) dependencies can be concluded alignment temperature in the air.

For the analysis of the influence of local intensive heat withdrawal on one of the boundaries to the thermal condition of the region in question is carried out the comparison of the values of mean air temperatures with the problem solution of the conjugate heat transfer in formulation (1) - (10) (Fig. 1) and with the uniform conditions on the border in the range of time $\tau=240000$ (Fig. 5).
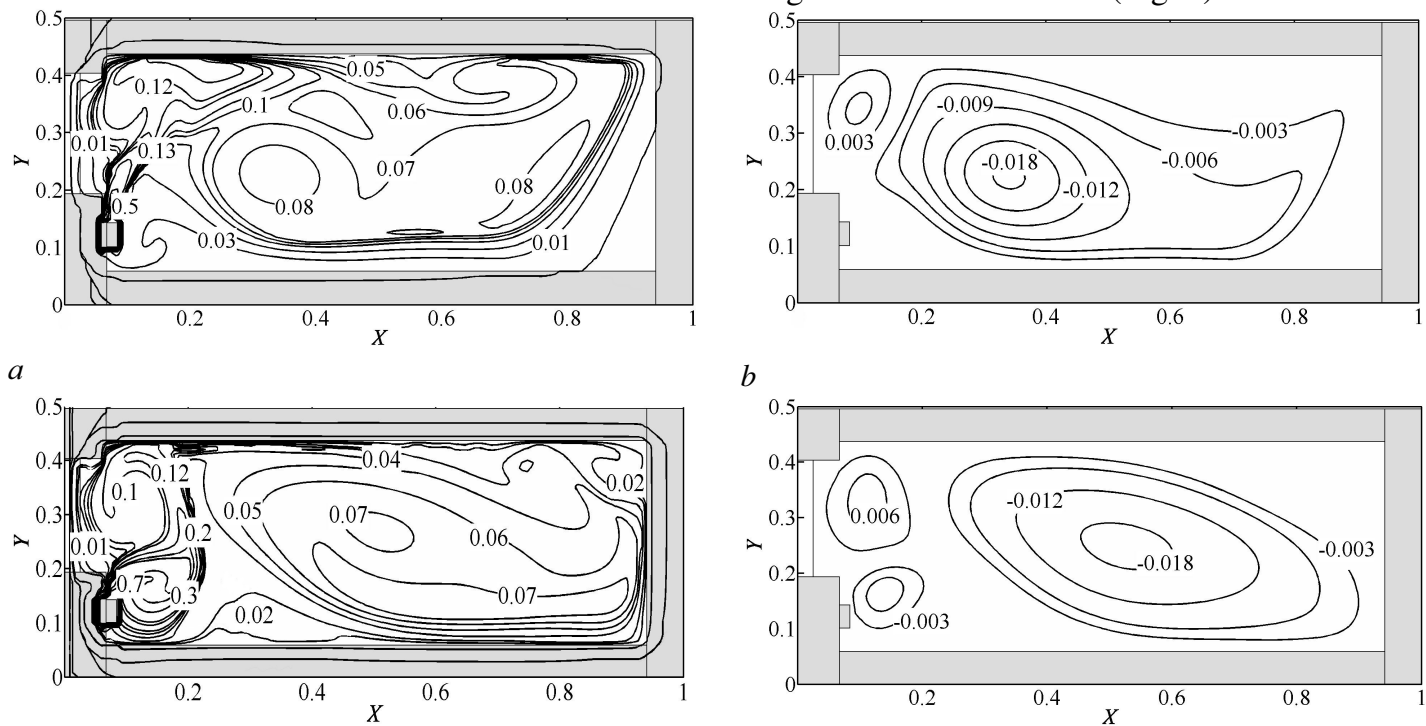

$c$

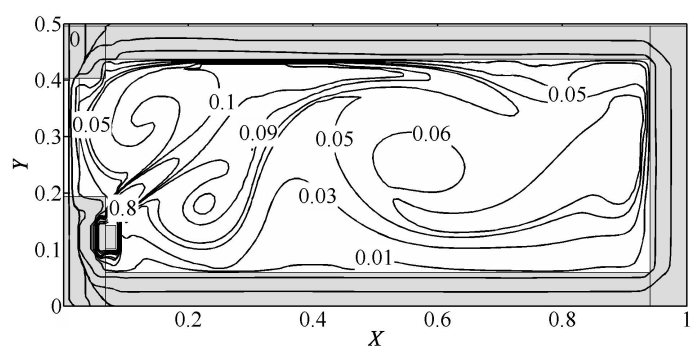

$d$

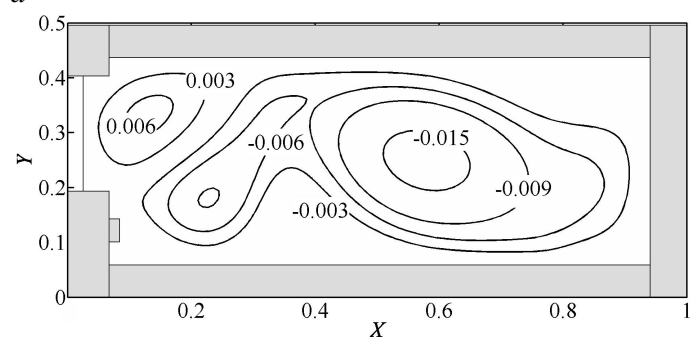

$e$
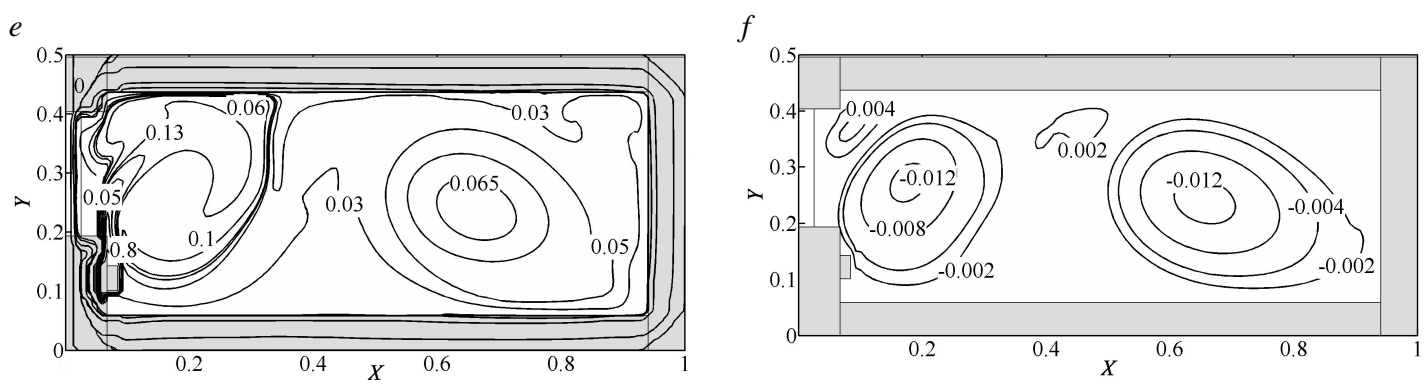

$g$

$h$

Figure 2. Area Temperature fields $(a, c, e, g)$ and the contours of stream function $(b, d, f, h)$ at different time $\tau: \tau$ $=20000(a, b), \tau=60000(c, d), \tau=90000(d, e), \tau=120000(g, h)$. 
TSOTR 2015

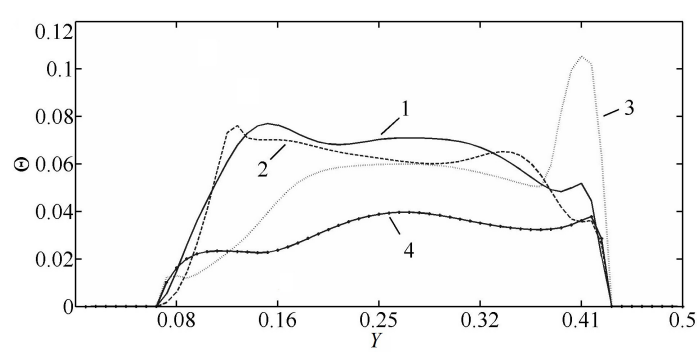

a

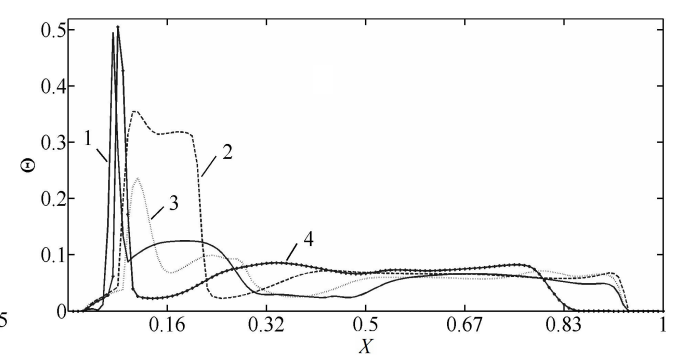

$b$

Figure 3. Temperature profile at sections $X=0.5(a)$ and $Y=0.5(b)$ at different $\tau: 1-\tau=20000,2-\tau=$ $60000,3-\tau=90000,4-\tau=120000$

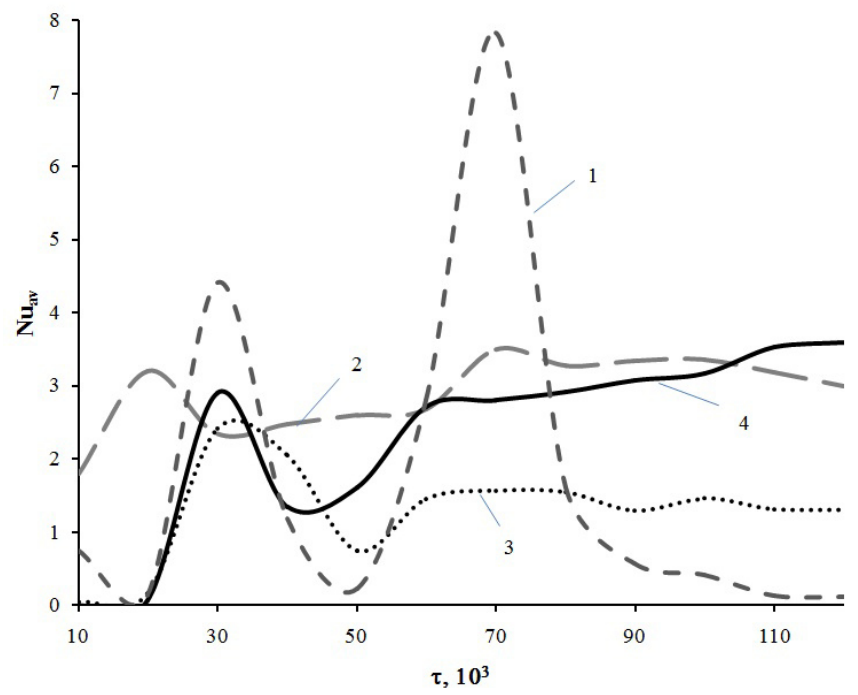

Figure 4. Dependence of the average Nusselt number on the time $\tau$ at interfaces: $1-X=L_{1} ; 2-Y=H_{U P} ; 3-Y=$ $H_{L B} ; 4-X=L_{R}$.

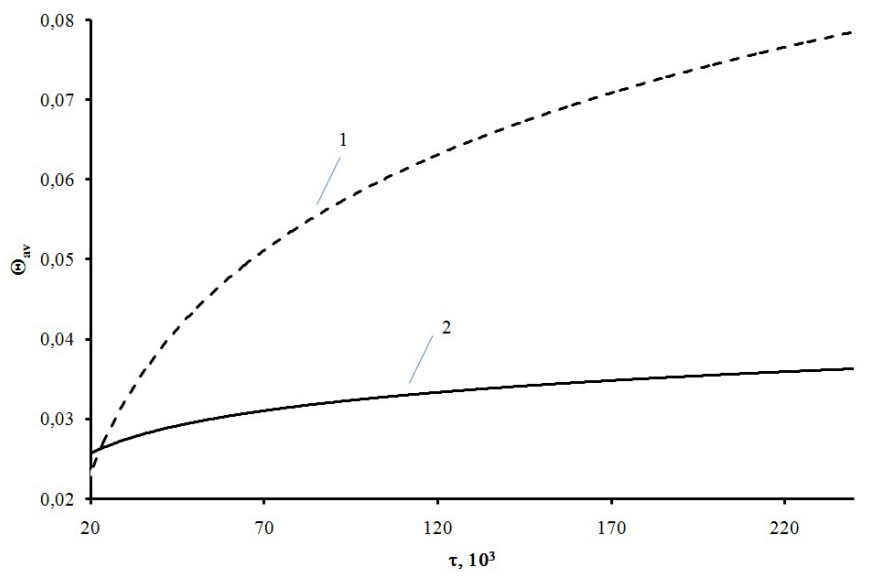

Figure 5. Values of the average temperature region of solutions (excluding the window aperture - 1, subject to the window aperture - 2). 
For the analysis of the influence of local intensive heat withdrawal on one of the boundaries to the thermal condition of the region in question is carried out the comparison of the values of mean air temperatures with the problem solution of the conjugate heat transfer in formulation (1) - (10) (Fig. 1) and with the uniform conditions on the border in the range of time $\tau=240000$ (Fig. 5).

\section{CONCLUSION}

Based on these results, we can conclude that the window aperture with a substantially inferior compared to the support structures under heat shield properties of the total area of the heat sink is not more than $10 \%$ significantly reduces the average temperature of the air (Fig. 5). In this previously established [2,3] laws of heat transfer in the considered fairly typical analysis in general remain. So it is possible to allocate a substantial unsteadiness of the formation of temperature fields due to the heat sink in enclosing structures and heat accumulation of materials these structures. It is also necessary to note the high gradients of temperatures in all characteristic zones of the region in the course of the prolonged (to $\tau=240000$ ) periods of time, which correspond to the physical (dimensional) time of 2 hours.

The work was realized within the research state assignment "Science" №13.1339.2014/K (Code of Federal Target Scientific and Technical Program 2.1410.2014).

\section{References}

1. Cengel Yu.A., Chajar A.J., Heat and Mass Transfer. Fundamentals and Applications, - 4th ed. New York : McGraw-Hill, 2011. - 905 p.

2. Kuznetsov G.V., Kurilenko N.I., Maksimov V.I., Mamontov G.Ya., Nagornova T.A., Heat transfer under heating of a local region of a large production area by gas infrared radiators, Journal of Engineering Physics and Thermophysics, 86 (2013), 3, pp. 519-524.

3. Maksimov V. I., Nagornova T.A., EPJ Web of Conferences, 76 (2014), Article number 01006.

4. Kuznetsov G.V., Sheremet M.A., New approach to the mathematical modeling of thermal regimes for electronic equipment, Russian Microelectronics, 37 (2008), 2, pp. 131-138.

5. Kuznetsov G.V., Sheremet M.A., Mathematical modelling of complex heat transfer in a rectangular enclosure, Thermophysics and Aeromechanics, 16 (2009), 1, pp. 119-128.

6. Belov I.A., Isaev S.A., Modeling of turbulent flows: Textbook. St. Petersburg.: Baltic State Technical University, 2001. 